\title{
Routing Algorithm of Wireless Sensor Network and Robustness Analysis Based on Fuzzy Mathematics
}

\author{
https://doi.org/10.3991/ijoe.v13i12.7888 \\ Ying Xiang $\left({ }^{\square}\right)$ \\ Changzhou Vocational Institute of Mechatronic Technology, Changzhou, China; \\ $570053025 @$ qq. com \\ Miaochao Chen \\ Chaohu University, Chaohu, China; \\ Xiaohong Zhuang \\ Changzhou Vocational Institute of Mechatronic Technology, Changzhou, China; \\ Xiaoxing $\mathrm{Li}$ \\ Future Land Holdings Co., Ltd, Shanghai, China
}

\begin{abstract}
In order to reduce the energy consumption and enhance the robustness of wireless sensor network (WSN), this paper proposes a hierarchical clustering routing algorithm based on fuzzy mathematics (HCRAFM). To make a comprehensive analysis of WSN, it is also necessary to detect the robustness of the network. Facing the multiple random variables, the traditional robustness detection models assume that all nodes have the same weight, making it impossible to quantify the analysis indices or obtain accurate results. Thus, the fuzzy mathematics theory was introduced to the WSN robustness detection, forming a fuzzy comprehensive evaluation method. The simulation results show that the HCRAFM strikes a load balance between WSN nodes, extends the life cycle of each node, and prolongs the service life of the network. In addition, the proposed algorithm is proved to have sound robustness and strong applicability.
\end{abstract}

Keywords-Routing Algorithms; Robustness Analysis; Wireless Sensor Network (WSN); Fuzzy Mathematics

\section{$1 \quad$ Introduction}

In wireless sensor network (WSN), the routing algorithm is responsible for setting up the path and mechanism of data transmission, realizing the dynamical update of network topology, and maintaining the information of network routing [1]. Therefore, the algorithm must be able to improve the energy efficiency of WSN nodes, and provide high quality services [2]. The traditional routing algorithms cannot satisfy the demand of WSN, which carries numerous different features from those of traditional networks. This calls for the design of a routing algorithm targeted at the demand of WSN [3]. The structure of a typical WSN is presented in Figure 1. 


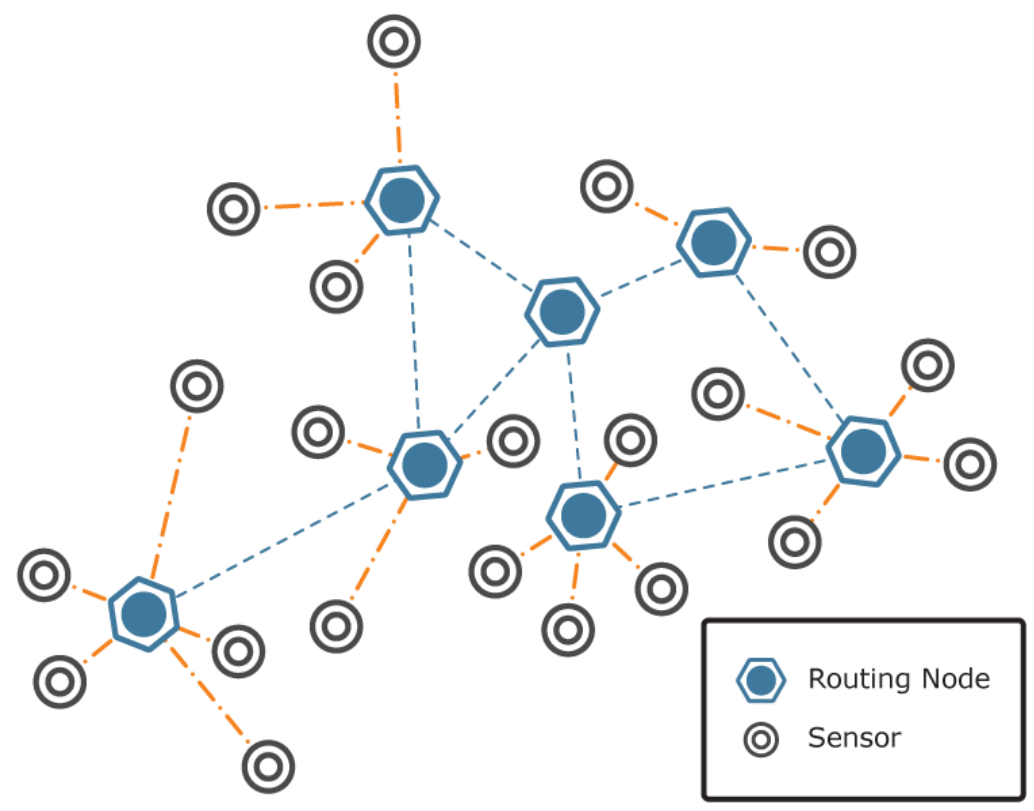

Fig. 1. Structure of a typical WSN

One of the most popular routing algorithm is clustering routing algorithm. The algorithm divides the network nodes into several clusters according to certain rules, such that each cluster contains a cluster head and a plurality of member nodes [4]. The overall closeness between cluster head and member nodes serves as the hallmark feature of the corresponding cluster [5]. The cluster head is selected from all nodes in the cluster by evaluating the residual energy of each node. In each round of computation, the selection of cluster head and the construction of routing topology consume an even shorter time than the stabilization of data acquisition and transmission.

The clustering routing algorithm supports both single-hop routing with one cluster head and multi-hop routing with several cluster heads. With direct data transmission between the cluster head and other network nodes, the single-hop routing does not require lots of information on the routing table [6][7] or much node storage space for network expansion. In this case, the network scalability is positively proportional to the speed of the cluster head.

By contrast, the multi-hop routing guarantees the energy balance in the network [8], because the network nodes use a less energy-consuming free space model thanks to the multi-path transmission channel between the cluster heads. However, the multipath transmission channel, together with the maintenance of routing table information, impose a high requirement on the performance of network nodes [9]. Fortunately, the performance of sensor nodes has been boosted substantially by the recent development of wireless sensor nodes, making it possible to popularize the application of multi-hop routing [10]. Figure 2 shows a classical multi-hop clustering routing algorithm. 


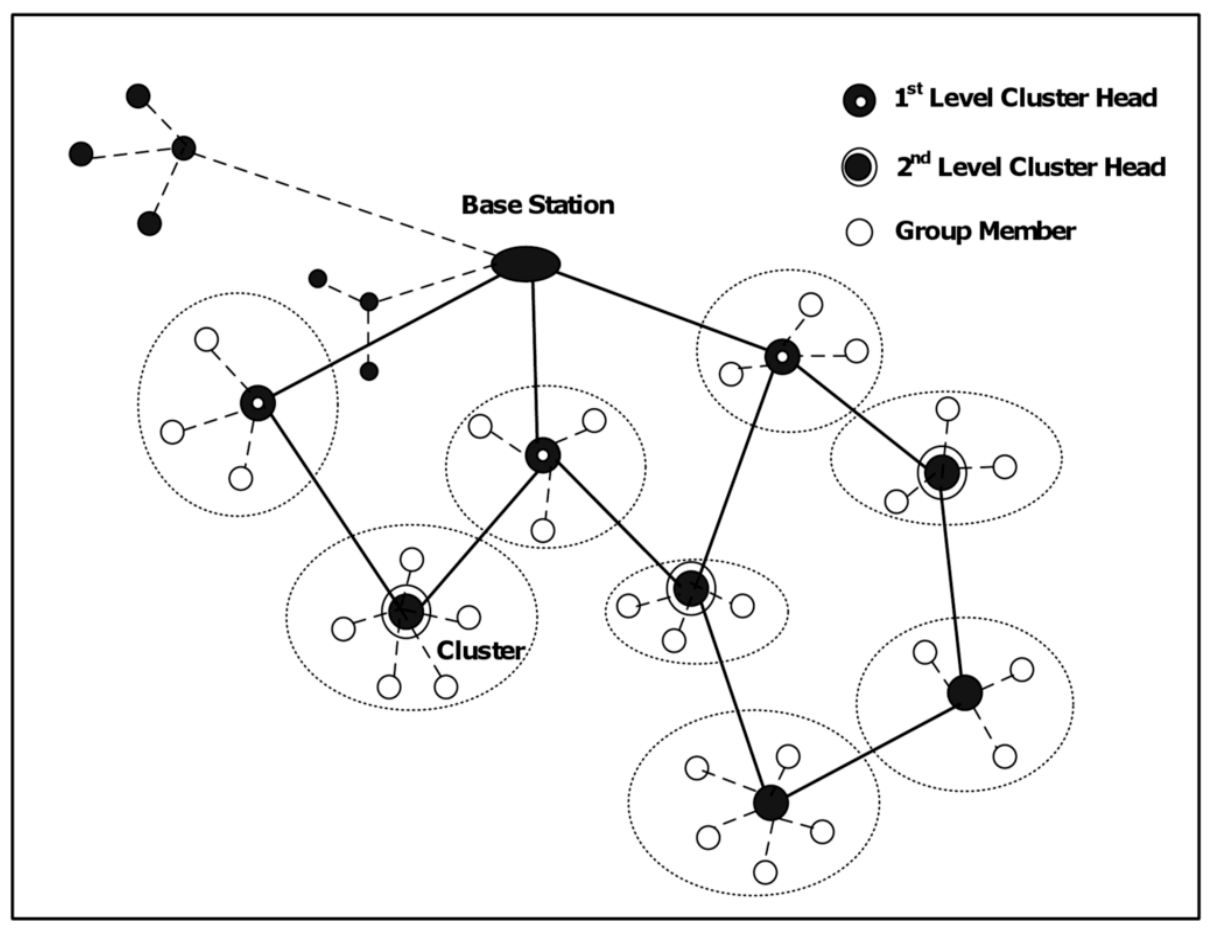

Fig. 2. Classical multi-hop clustering routing algorithm

As its name suggests, the WSN is a wireless network consisting of numerous sensor nodes with sensing, computing and communication functions. The network has been extensively applied to smart cities, precision agriculture, environmental monitoring and many other emerging fields. Since the sensor nodes are usually powered by a limited number of batteries, it is a hot topic and key point to reduce the energy consumption of WSN.

Proper routing is one of the most effective ways to lower the energy demand of WSN. Several clustering routing algorithms have been developed for this purpose. The first of this kind is the low energy adaptive clustering hierarchy (LEACH) algorithm, in which all nodes share the same probability of becoming cluster head. Thus, the energy consumption is evenly distributed across all nodes in the network [11]. Nonetheless, this algorithm also has a downside: the cluster heads are in single-hop mode and communicate with the base station (BS), so that the nodes far away from the BS inevitably consume an excessive amount of energy.

The drawback can be resolved by integrating multi-hop routing and the hybrid energy efficient distributed (HEED) algorithm. Considering both the primary and secondary parameters, the HEED can select cluster heads with uniform communication load and thus balance the load in the network by selecting cluster heads with uniform load. The multi-hop routing consumes less energy than the single-hop routing in the communication with the BS [12]. In this scenario, however, the clusters near the BS 
demand greater energy to forward the huge amount of head information from remote clusters [13].

To solve the above problems, some non-uniform clustering methods have been invented, namely the energy-efficient uneven clustering (EEUC) algorithm, the distributed energy-balanced unequal clustering routing protocol (DEBUC), and the uneven clustering routing protocol based on dynamic partition (UCDP). These methods reduce data forwarding tasks, shorten the communication radius of near-BS clusters, thereby improving the load balance of the whole network.

From single-hop clustering to multi-hop routing, and then to non-uniform clustering, the research into WSN energy conservation has become increasingly extensive and in-depth. However, there is still great room for improving the rationality of clustering, especially cluster head communication, without obvious increase in computing load [14]. For instance, the hierarchical design model can be introduced to consider the energy consumption, density and centrality of nodes prior to selecting cluster head. After the selection, it is wise to adopt multi-hop routing for cluster head communication. These moves are likely to further balance node load and slash the energy consumption of the network. If there is no complicated mathematical modelling, the fuzzy algorithm can be employed to select cluster heads, as it is able to prioritize some factors after evaluating the multiple factors of WSN.

In light of the above, this paper proposes a hierarchical clustering routing algorithm based on fuzzy mathematics (HCRAFM) for WSN. First, the network was divided into multiple layers based on the distance to the BS. In each layer, the number of cluster heads is negatively correlated with the cluster-BS spacing. Then, the cluster heads were selected by fuzzy algorithm, considering the residual energy, density and centrality of nodes. Finally, the cluster heads transmitted data to establish a multi-hop routing with the BS. The proposed algorithm can balance network load and reduce energy consumption without pushing up the computing complexity.

\section{HCRAFM}

\subsection{WSN and energy model}

WSN. The HCRAFM makes the following assumptions for WSN: First, the nodes are randomly distributed in the detection area, all of which share the same initial energy [16]; Second, each node can adjust its transmission load according to the need, and learn about its distance from other nodes through received signal strength indicator (RSSI); Third, all nodes have enough computing power to complete the algorithm.

Energy model. The free space model and the multipath fading model were adopted according to the distance between the sending node and the receiving node. Assuming that the sending node needs to transmit $k$ bit data to the receiving node over the distance $d$, the sending energy $E_{t}$ consumed by the sending node and the receiving energy $E_{r}$ consumed by the receiving node can be respectively expressed as: 


$$
\begin{gathered}
E_{t}=\left\{\begin{array}{c}
\left(E_{\text {elec }}+d^{2} \cdot E_{\text {amp } 1}\right) \cdot k, d<d_{0} \\
\left(E_{\text {elec }}+d^{4} \cdot E_{\text {amp } 2}\right) \cdot k, d \geq d_{0}
\end{array}\right. \\
E_{r}=k \cdot E_{\text {elec }}
\end{gathered}
$$

where $E_{\text {elec }}$ is the energy consumption of the transceiver; $E_{\text {amp } 1}$ and $E_{\text {amp } 2}$ are the energy consumption of the amplifier in the free space model and multipath fading model, respectively. The two models should be selected based on the communication distance $d_{0}$.

\subsection{Network stratification}

The roulette strategy was employed in the HCRAFM. Each round is implemented in the following steps. First, the network was divided into several layers based on the relationship between energy and communication distance. Then, the layer of each node was determined according to the distance from the BS. Finally, the number of cluster heads in each layer was identified depending on the number of nodes in the layer and the distance of the layer from the BS [17]. The shorter distance between the layer and the BS means that its cluster is much closer to the BS.

The purpose of the HCRAFM is to reduce the data forwarding tasks of near-BS sensor nodes and achieve the load balance across the network. Considering the three variables (node energy, density and centrality) of each layer, multiple clusters were selected by fuzzy algorithm. The cluster heads did not die prematurely, and the intracluster communication consumed less energy. The other nodes joined the clusters formed around the nearest cluster head. The intra-cluster communication followed the single-hop mode, while the inter-layer communication was completed via a multi-hop routing between the cluster heads and the BS. Thus, there was no long-distance transmission of signals [18].

According to the residual energy of cluster heads and the time of each round, the cluster heads were re-selected and a new route was established to maintain the routing.
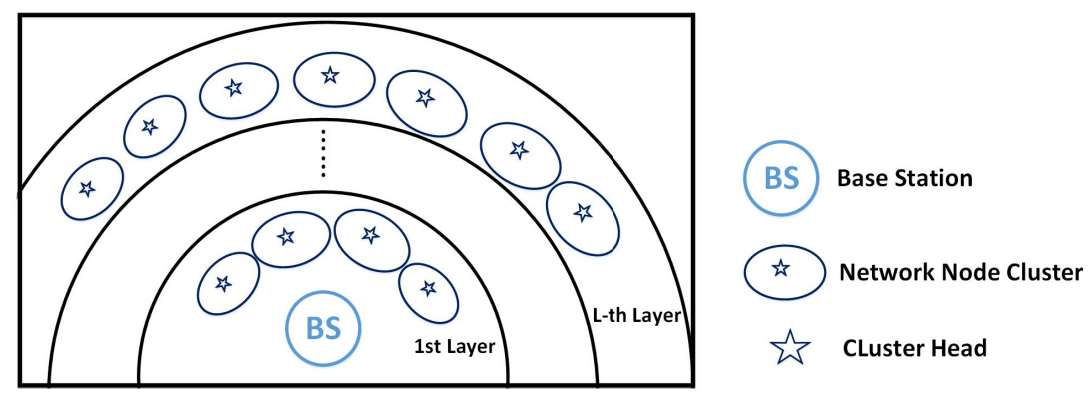

Fig. 3. Network stratification 
As shown in Figure 3, the network has been divided to L layers based on the $d_{0}$ in the energy model. Let $d$ be the distance from a node to the BS. Then, the maximum node-BS distance $D_{\max }$ is:

$$
\begin{gathered}
d_{\max }=\operatorname{MAX}\left(d\left(S N_{j}, B S\right)\right) \\
L=\left[2 \times \frac{d_{\max }}{d_{0}}\right]
\end{gathered}
$$

The sensor node $S N_{j}$ belongs to the number of the $l$-th layer,

$$
l=\left[2 \times \frac{d_{j}}{d_{0}}\right]
$$

In this way, the communication distance between cluster heads and layers became shorter than $d_{0}$, thus lowering the energy consumed in remote communication. The number of cluster heads in each layer is inversely proportional to the number of layers $\mathrm{L}$, and positively proportional to the number of nodes $N_{l}$ in the $l$-th layer. Thus, both the number of cluster heads $C l$ in the near-BS layer and the energy consumption of intra-cluster communication exhibited a decreasing trend. The $\mathrm{Cl}$ is calculated as follows:

$$
C l=K \times \frac{N_{l}}{l}
$$

\subsection{Cluster head selection}

The fuzzy algorithm has the advantages of simple structure and good robustness, without needing to go through complicated mathematical modelling [8][9]. Fuzzy variables and rules were applied to the sensor network layer of each layer, and the nodes prioritized by fuzzy algorithm were selected as cluster heads.

Fuzzy variables.

Relative energy. The relative energy $R E$ is the ratio the residual energy $E_{i}$ of node $S N_{i}$ to the maximum residual energy $E_{\max }$.

$$
R E=\frac{E_{i}}{E_{\max }}
$$


Relative density. The node density refers to the density of nodes in the vicinity of a node. It is expressed as the number $D_{i}$ of neighbouring nodes in the communication range $d_{0}$ of node $S N_{i}$. The higher the density, the less energy is needed to transmit data from the node to the neighbouring nodes. The relative density $R D$ is the ratio of the density $D_{i}$ of node $S N_{i}$ to the maximum density $D_{\max }$ of the nodes in the cluster of node $S N_{i}$.

$$
R D=\frac{D_{i}}{D_{\max }}
$$

Relative centrality. The node centrality $C_{i}$ describes the proximity of node $S N_{i}$ to its neighbouring nodes. It is expressed as the formula below.

$$
C_{i}=\sqrt{\left(x_{i}-\frac{1}{n} \sum_{j=1}^{n} x_{j}\right)^{2}+\left(y_{i}-\frac{1}{n} \sum_{j=1}^{n} y_{j}\right)^{2}}
$$

where $x_{i}$ and $y_{i}$ are respectively the $\mathrm{x}$ - and $\mathrm{y}$ - coordinates of node $S N_{i}$. The higher the centrality, the less energy is needed to transmit data from the node to the neighbouring nodes. The relative centrality $R C$ is the ratio of the centrality $C_{i}$ of node $S N_{i}$ to the minimum centrality $C_{\min }$ of the nodes in the cluster of node $S N_{i}$.

$$
R C=\frac{C_{\min }}{C_{i}}
$$

Fuzzification of variables. The fuzzification of variables is to map each input value to the corresponding fuzzy set and assign membership functions to each fuzzy set [10]. Assuming that the relative energy $R E_{i}=e^{*}$, relative density $R D_{i}=d^{*}$ and relative centrality $R C_{i}=c^{*}$, the corresponding fuzzy inputs $E^{*}(e), D^{*}(d)$ and $C^{*}(d)$ can be represented as:

$$
\begin{gathered}
E^{*}(e)=\left\{\begin{array}{l}
1, \mathrm{e}=\mathrm{e}^{*} \\
0, \mathrm{e} \neq \mathrm{e}^{*}
\end{array}\right. \\
D^{*}(d)=\left\{\begin{array}{l}
1, d=d^{*} \\
0, d \neq d^{*}
\end{array}\right.
\end{gathered}
$$




$$
C^{*}(d)=\left\{\begin{array}{l}
1, c=c^{*} \\
0, c \neq c^{*}
\end{array}\right.
$$

Triangular and trapezoidal membership functions were adopted because of their applicability to real-time computing. Based on the 3 fuzzy input variables, i.e., relative energy, relative density and relative centrality [17], the HCRAFM outputted an output variable: node priority. The output variable depicts whether a node has been selected as a cluster head.

By empirical experience, the fuzzy subsets and fuzzy membership functions were established corresponding to the input and output variables. The resulting variables and fuzzy sets are listed in Table 1.

Table 1. Parameters and feature set

\begin{tabular}{|l|l|}
\hline \multicolumn{1}{|c|}{ Parameter } & \multicolumn{1}{c|}{ Feature set } \\
\hline Relative energy(RE) & Less,medium,ormore \\
\hline Relative density(RD) & Low,medium,tall \\
\hline Relative centrality(RC) & Low,medium,tall \\
\hline Node priority(Sup) & Very low,low,slightly low,medium,slightly high, high, very high \\
\hline
\end{tabular}

Figures 4 and 5 detail the fuzzy relationships between these variables and the membership degree. Figure 4(a) illustrates how the relative energy varies with the membership degree. The relative energy was an eigenvalue when the membership degree fell in a certain range [3]. The correlation between the membership degree and relative energy remains on the low level when the relative energy was less than 0.2. And it enters the middle level when the relative energy is between 0.3 and 0.7 , and climbs up to the high level when the relative energy exceeds 0.8 . When the relative energy falls between 0.2 and 0.3 , the correlation between the membership degree and relative energy belonged to both the low and middle levels. When the relative energy varies between 0.7 and 0.8 , the correlation belongs to the middle and high levels at the same time. Figures 4(b), 5(a) and 5(b) depict the relationships between membership degree and the relative density, relative centrality and node priority, respectively. These relationships are similar to the relationship between membership degree and relative energy. 


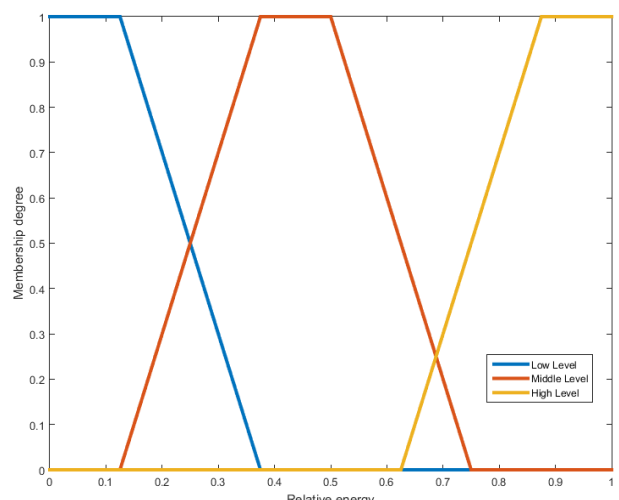

(a) Relative energy vs. Membership degree

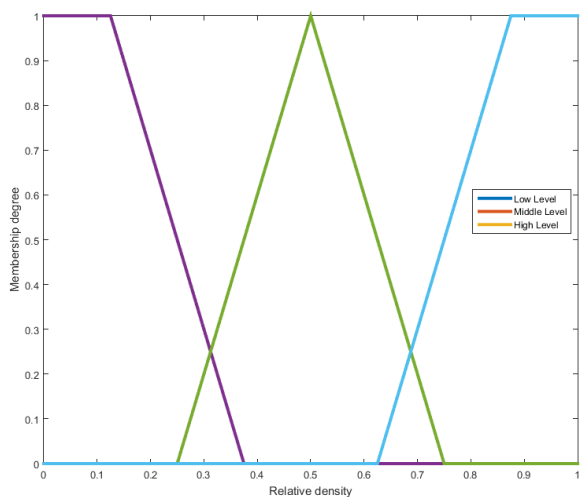

(b) Relative density vs. Membership degree

Fig. 4. Relative energy and relative density vs. membership degree

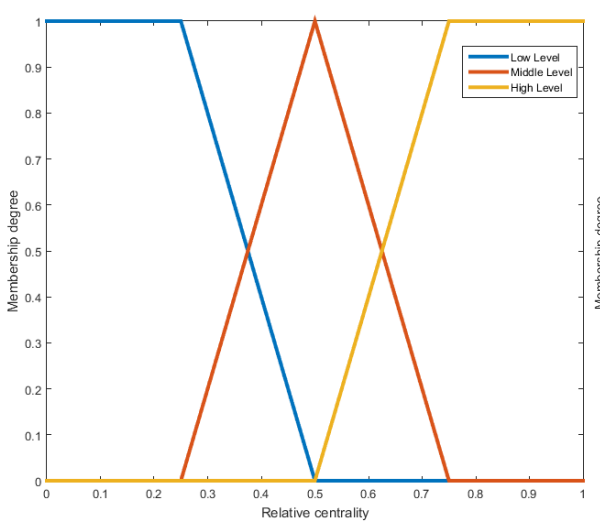

(a) Relative centrality vs. Membership degree

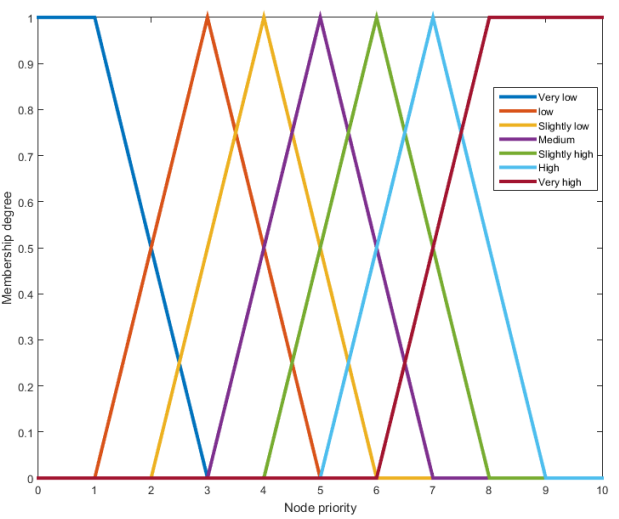

(b) Node priority vs. Membership degree

Fig. 5. Relative centrality and node priority vs. membership degree

Fuzzy rules. The HCRAFM uses fuzzy If-Then classification rules. If the node has high relatively energy, high relatively density and high relatively centrality, then the node priority must be very high [2]. As shown in Table 2 below, the HCRAFM contains $3 \times 3 \times 3=27$ fuzzy rules. 
Table 2. Fuzzy rules

\begin{tabular}{|c|c|c|c|c|}
\hline Rules & Relative energy & Relative density & Relative centrality & Node priority \\
\hline 1 & Less & Low & Low & Very Low \\
\hline 2 & Less & Low & Middle & Very Low \\
\hline 3 & Less & Low & High & Very Low \\
\hline 4 & Less & Middle & Low & Very Low \\
\hline 5 & Less & Middle & Middle & Very Low \\
\hline 6 & Less & Middle & High & Very Low \\
\hline 7 & Less & High & Low & Very Low \\
\hline 8 & Less & High & Middle & Very Low \\
\hline 9 & Less & High & High & Very Low \\
\hline 10 & Medium & Low & Low & Slightly Low \\
\hline 11 & Medium & Low & Middle & Slightly Low \\
\hline 12 & Medium & Low & High & Middle \\
\hline 13 & Medium & Middle & Low & Slightly Low \\
\hline 14 & Medium & Middle & Middle & Middle \\
\hline 15 & Medium & Middle & High & Slightly High \\
\hline 16 & Medium & High & Low & Slightly Low \\
\hline 17 & Medium & High & Middle & Middle \\
\hline 18 & Medium & High & High & Slightly High \\
\hline 19 & More & Low & Low & Slightly Low \\
\hline 20 & More & Low & Middle & Middle \\
\hline 21 & More & Low & High & Slightly High \\
\hline 22 & More & Middle & Low & Middle \\
\hline 23 & More & Middle & Middle & Slightly High \\
\hline 24 & More & Middle & High & High \\
\hline 25 & More & High & Low & Middle \\
\hline 26 & More & High & Middle & High \\
\hline 27 & More & High & High & Very high \\
\hline
\end{tabular}

Defuzzification. Defuzzification is the transformation of the output generated by fuzzy rules into practical variables [7]. The centroid point method was adopted for defuzzification. The process is expressed as follows:

$$
\sup =\frac{\int x \cdot \mu_{\text {sup }}(x) d x}{\int \mu_{\text {sup }}(x) d x}
$$

where Sup is node priority; $\sup (x)$ is the membership function of the fuzzy set for the node priority; $x$ is the specific value of node priority. The relative energy, density and centrality of set node $S N_{i}$ are $\mathrm{PE}=0.765, \mathrm{PD}=0.5$ and $\mathrm{PC}=0.5$. 
Here we set the relative energy, relative density and relative centrality of $S N_{i}$ to $R E=0.765, R D=0.5, R C=0.5$. Thus, the relative energy falls into the middle level when the membership degree is $\mu_{3}=0.325$, and it falls into the high level when the membership degree is $\mu_{4}=0.175$. The relative density and the relative centrality belong to middle level when the membership degree is equal to 1 . The node priority stays on the middle level when the membership degree was $\mu_{3}=0.325$, and the high level when the membership degree is $\mu_{4}=0.175$.

Therefore, the value of the priority of the $S N_{i}$ node is:

$$
\sup =\frac{\sup _{m i d} \times \mu_{3}^{*}+\sup _{h i g h} \times \mu_{4}^{*}}{\mu_{3}^{*}+\mu_{4}^{*}}=5.73
$$

\subsection{Clustering and routing}

Once a node was selected as a cluster head, it would broadcast information to its neighbouring nodes. Upon receiving the information, each neighbouring node would join the cluster of the nearest cluster head, and transmit the information to the cluster head. The purpose is to minimize the cost of the communication between nodes and cluster heads [13].Then, the cluster head would collect, process and send the information to the BS. The energy consumption of data fusion is denoted as EDA.

The inter-layer routing is shown in Figure 6. In the figure, $\mathrm{CH}_{l i}$ is the cluster head of the $l$-th layer. The data to be transmitted to the BS was forwarded by this layer to the nearest cluster head $\mathrm{CH}_{l-1}$ of $l-1$-th layer. Then, the cluster head $\mathrm{CH}_{l-1}$ would forward the data to the BS. After that, the BS would transmit these data to the nearest cluster head $\mathrm{CH}_{l-2}$ of $\mathrm{l}-2$ layer. The cluster head $\mathrm{CH}_{1 p}$ of each layer would transmit data to the BS in turns until that of the first layer completed the transmission. At this moment, the multi-hop routing of data was established between the layers and the BS.

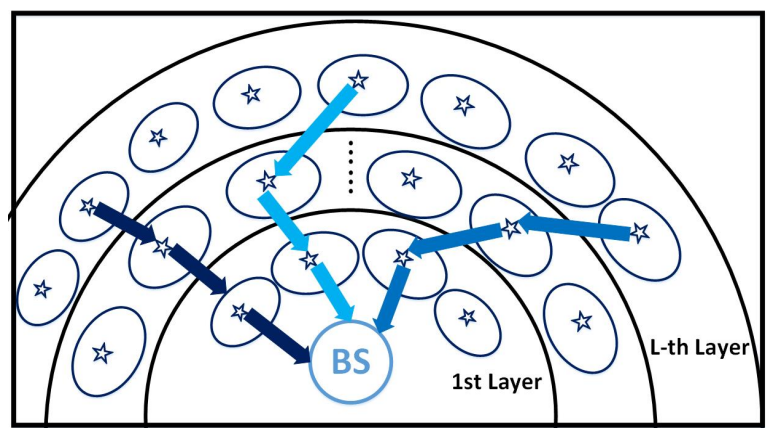

Fig. 6. Multilayer wireless routing 
If the energy of a cluster head in the $l$-th layer was less than half of the average residual energy in the cluster, a new cluster head should be selected and a new cluster should be established within the $l$-th layer. Next, a new path would be established to forward the data of the cluster head from the $1^{\text {st }}$ layer to the $l$-th layer. After a time of $\mathrm{T}$ has elapsed, the entire network would start a new round of routing.

\section{Robustness Evaluation}

\subsection{Overview}

The robustness of WSN means the communication performance of the network is not affected by random external interference under normal conditions. During the robustness evaluation of WSN, the quantitative analysis was transformed into qualitative membership principle of fuzzy mathematics. The difference nodes were analysed through inference.

The HCRAFM is very accurate and effective in handling the fuzzy issues that are hard to quantify [5]. In view of the difficulties in quantify the qualitative variables of WSN with mathematical language, the robustness of the network was roughly categorized into four levels in the evaluation: extra-high, high, middle and low.

Whereas neither qualitative method or general data analysis can obtain the accurate robustness of WSN, the comprehensive analysis of fuzzy mathematics is capable of measure the exact robustness of the network through detailed discussion of various factors [8], making it possible to acquire quantitative analysis results.

Featuring profound fuzziness, the information vector exists as a fuzzy subset with no specific value. Thus, it is an ideal tool to depict the robustness of the network. The robustness of WSN can be analysed accurately and objectively through fuzzy comprehensive analysis [10].

\subsection{Implementation}

Step 1: The robustness of the procedure was explicitly determined by the robustness of the target area $U=\left\{U_{1}, U_{2}, \mathrm{~L}, U_{i}, \mathrm{~L}, U_{m}\right\}$, which is a set of comprehensive evaluation indices. The $i$-th level indices are denoted as $U_{i}=\left\{U_{i 1}, U_{i 2}, \mathrm{~L}, U_{i j}, \mathrm{~L}, U_{i n}\right\}$ and the indices on both the $i$-th and $j$-th levels are denoted as $U_{i j}$.

Step 2: The comment set is denoted as V, which is expressed as $V=\left\{V_{1}, V_{2}, \mathrm{~L}, V_{n}\right\}$. Different scores were assigned to the fuzzy subsets to depict the different levels.

Step 3: The factors were evaluated one by one. Based on the single-factor analysis, the fuzzy relation matrix $R$ was constructed through the normative analysis based on 
the requirements of different factors, and the membership evaluation of fuzzy subsets of different scores.

$$
R=\left(\begin{array}{c}
R_{1} \\
R_{2} \\
\mathrm{M} \\
R_{m}
\end{array}\right)=\left(\begin{array}{cccc}
r_{11} & r_{12} & \mathrm{~L} & r_{1 n} \\
r_{21} & r_{22} & \mathrm{~L} & r_{2 m} \\
\mathrm{M} & \mathrm{M} & \mathrm{M} & \mathrm{M} \\
r_{m 1} & r_{m 2} & \mathrm{~L} & r_{m n}
\end{array}\right)
$$

The $\mathrm{R}$ matrix is responsible for depicting the relationship between the fuzzy relation matrix $\mathrm{U}$ and the comment set $\mathrm{V}$.

Step 4: The fuzzy weight vector of the evaluation factor was clearly defined as $\mathrm{A}=$ (A1, A2). This is because the single factors differ in the analysis function.

Step 5: The fusion operation was reasonably configured to fuse A with different metrics $\mathrm{R}$. The different metrics $\mathrm{R}$ were used to describe the analysis objects according to the membership degree of fuzzy subsets obtained in single factor analysis. After fusing different rows with the fuzzy weight vector, the author obtained the different metrics relative to the membership degree of fuzzy subsets of different scores. The vector is called the structural vector B for fuzzy comprehensive evaluation. Hence, the model is constructed as:

$$
\begin{aligned}
& B=A \mathrm{o} R=\left(b_{1}, b_{2} \mathrm{~L}, b_{n}\right) \\
& =\left(a_{1}, a_{2}, \mathrm{~L}, a_{m}\right)\left(\begin{array}{cccc}
r_{11} & r_{12} & \mathrm{~L} & r_{1 n} \\
r_{21} & r_{22} & \mathrm{~L} & r_{2 m} \\
\mathrm{M} & \mathrm{M} & \mathrm{M} & \mathrm{M} \\
r_{m 1} & r_{m 2} & \mathrm{~L} & r_{m n}
\end{array}\right)
\end{aligned}
$$

The weighted average model was introduced to describe the accurate value of different factors, that is, the weighted average operator was adopted for the fuzzy comprehensive evaluation.

\section{Simulation}

\subsection{Simulation settings}

In the MATLAB simulation, the HCRAFM was contrasted with the LEACH and the EECU. A total of 400 wireless sensor nodes were deployed within a $200 * 200 \mathrm{~m}^{2}$ area. The BS station was arranged at $(100,-50) \mathrm{m}$, and the initial energy of each node was set to $0.5 \mathrm{~J}$. The other parameters are shown in Table 3 . The performance of these algorithms was analysed under selective interference. 
Paper-Routing Algorithm of Wireless Sensor Network and Robustness Analysis Based on Fuzzy Mat..

Table 3. Simulation parameters

\begin{tabular}{|c|c|}
\hline Parameters & Values \\
\hline Network coverage area & $(0,0) \sim(200,200) \mathrm{m}$ \\
\hline Node number & 400 \\
\hline Base station location & $(100,-50) \mathrm{m}$ \\
\hline Initial energy of node & $0.5 \mathrm{~J}$ \\
\hline$d_{o}$ & $87 \mathrm{~m}$ \\
\hline$E_{\text {elec }}$ & $50 \mathrm{~nJ} / \mathrm{bit}$ \\
\hline$E_{\text {ampl }}$ & $10 \mathrm{pJ} / \mathrm{bit} / \mathrm{m} 2$ \\
\hline$E_{\text {amp } 2}$ & $0.0013 \mathrm{pJ} / \mathrm{bit} / \mathrm{m} 4$ \\
\hline$E_{D A}$ & $5 \mathrm{~nJ} /(\mathrm{bit} . \mathrm{signal})$ \\
\hline$T$ & $1 \mathrm{~s}$ \\
\hline Packet length & $4000 \mathrm{bit}$ \\
\hline
\end{tabular}

\subsection{Fuzzy mathematics model}

According to the simulation results in Figure 7, the node priority was small when the relative energy was 0 , regardless of the relative centrality or relative density. When the relative energy reached a certain value, the node priority increased with the relative density and relative centrality. The trend indicates that the relative energy is the primary influencing factor on node priority. Of course, the relative density and centrality also have a significant impact on the node priority.

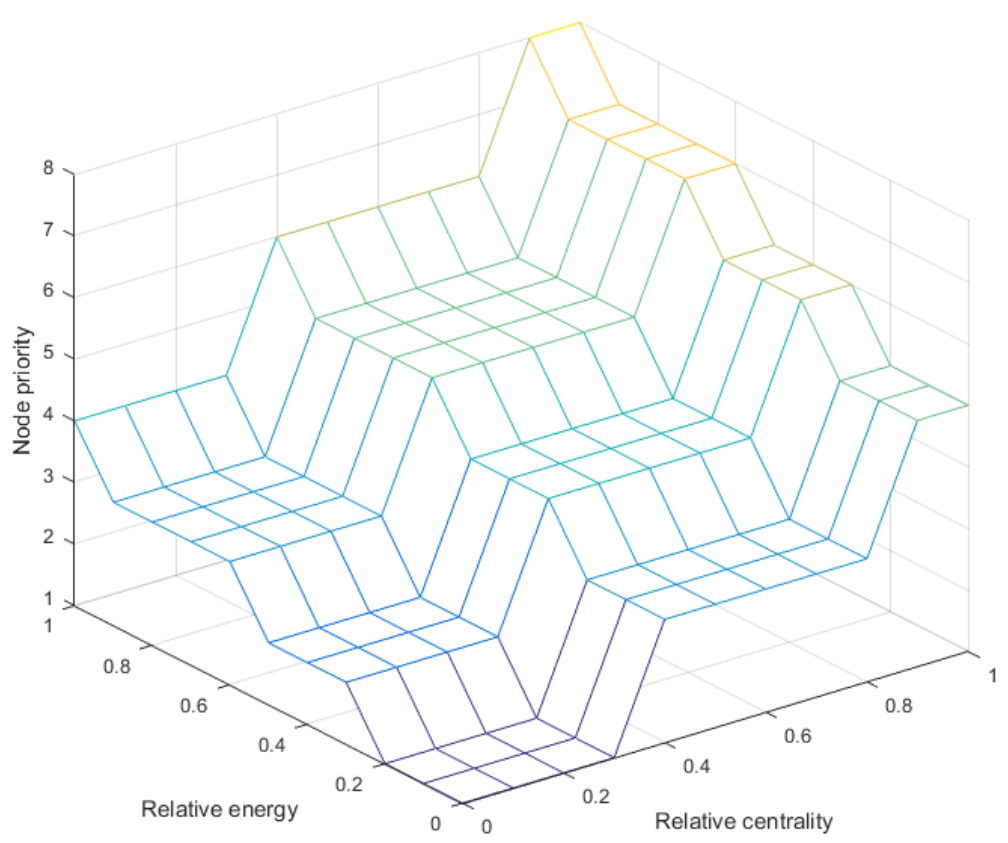

Fig. 7. Simulation results of fuzzy mathematics model 


\subsection{Energy consumption of cluster head}

This section compares the cluster head energy consumption curves obtained by the HCRAFM, the LEACH and the EEUC. The energy consumption in the first $20 \mathrm{~s}$ is recorded in Figure 8. It can be seen that the energy consumptions calculated by the HCRAFM and the EEUC were smaller and more stable than that obtained by the LEACH. A possible reason lies in the different selection methods of cluster head in these algorithms. The LEACH chooses cluster head randomly by voting, resulting in significant fluctuation in the energy consumption of cluster head. The HCRAFM and the EEUC select cluster head more stably under stricter conditions. Besides, the two algorithms also save more energy than the LEACH, thanks to the adoption of the multi-hop routing. The cluster head energy consumption is further reduced in the HCRAFM due to the integration of the fuzzy mathematics algorithm, which considers the energy and location information of cluster heads. Therefore, the HCRAFM algorithm achieved the most balanced energy consumption effect.

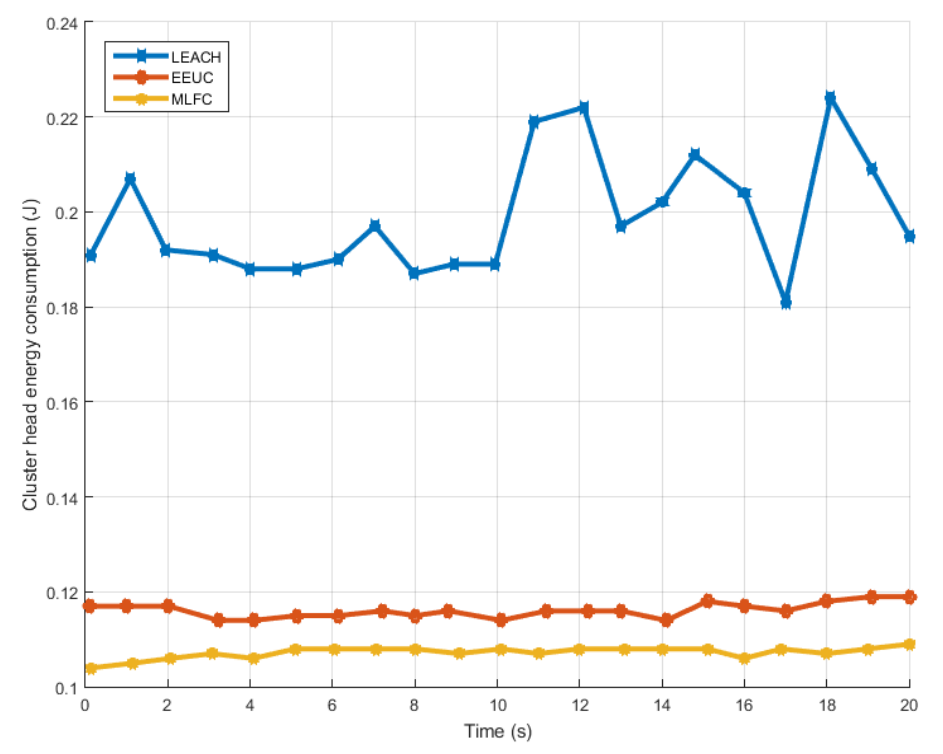

Fig. 8. Energy consumption curves of cluster heads with different algorithms

\subsection{Network energy consumption}

This section compares the network energy consumption curves obtained by the HCRAFM, the LEACH and the EEUC. As shown in Figure 9, the HCRAFM yielded lower network energy consumption than the two contrastive algorithms. The performance edge is attributable to the adoption of the fuzzy mathematics theory, which considers factors like node energy, centrality and density, the decrease in intra-cluster energy consumption resulted from rational selection of cluster heads, and the decline in extra-cluster energy consumption caused by the multi-hop routing. 


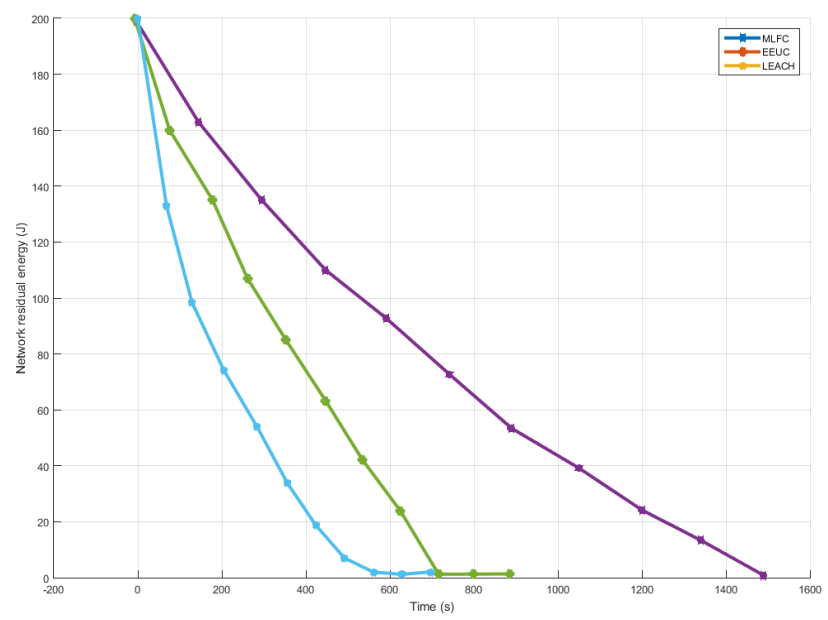

Fig. 9. Network energy consumption curves with different algorithms

\subsection{System robustness analysis}

Under the same selective interference, this section analyses the robustness of the network obtained by the HCRAFM and the traditional equal weight method. According to the results in Figure 10, the HCRAFM achieved much better network robustness than the traditional method with the increase in the number of network interference nodes, indicating that it is an efficient WSN robustness detection method. This is because the fuzzy mathematics method can accurately analyse the selective interference factors of the network, and, in turn, detect the exact robustness of the network.

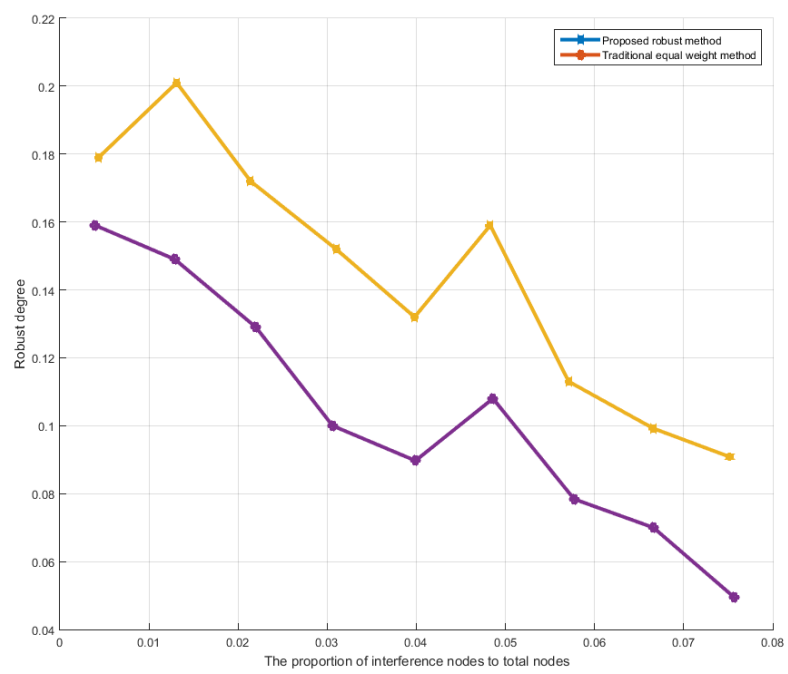

Fig. 10. System robustness analysis results 


\section{Conclusions}

This paper puts forward the HCRAFM to reduce the energy consumption and improve the robustness of WSN. The proposed algorithm first divides the network into several layers, then selects multiple cluster heads using the fuzzy algorithm based on node energy, density and centrality in each layer. Then, the residual nodes joined the cluster of the nearest cluster head in the same layer, which transmitted data and established multi-hop routing by themselves. To make a comprehensive analysis of WSN, it is also necessary to detect the robustness of the network. Facing the multiple random variables, the traditional robustness detection models assume that all nodes have the same weight, making it impossible to quantify the analysis indices or obtain accurate results. Thus, the fuzzy mathematics theory was introduced to the WSN robustness detection, forming a fuzzy comprehensive evaluation method. The simulation results show that the HCRAFM strikes a load balance between WSN nodes, extends the life cycle of each node, and prolongs the service life of the network. In addition, the proposed algorithm is proved to have sound robustness and strong applicability.

\section{Acknowledgment}

This work is supported bythe University Natural Science Key Foundation of Anhui (No. KJ2017A453), the University Teaching Research Foundation of Anhui (No. 2016 jyxm0693).

\section{$7 \quad$ References}

[1] Han, G., Jiang, J., Shu, L., Niu, J., \& Chao, H. C. (2014). "Management and applications of trust in Wireless Sensor Networks: A survey." Journal of Computer and System Sciences, 80(3): 602-617. https://doi.org/10.1016/j.jcss.2013.06.014

[2] Shamshirband, S., Amini, A., Anuar, N. B., Kiah, M. L. M., Teh, Y. W., \& Furnell. (2014). "D-FICCA: A density-based fuzzy imperialist competitive clustering algorithm for intrusion detection in wireless sensor networks." Measurement, 55: 212-226. https://doi.org/10.1016/j.measurement.2014.04.034

[3] Oda, T., Elmazi, D., Barolli, A., Sakamoto, S., Barolli, L., \& Xhafa, F. (2016). "A genetic algorithm-based system for wireless mesh networks: analysis of system data considering different routing protocols and architectures." Soft Computing, 20(7): 2627-2640. https://doi.org/10.1007/s00500-015-1663-Z

[4] Guo, W.J., Zhang, W. (2014) "A survey on intelligent routing protocols in wireless sensor networks." Journal of Network and Computer Applications, 38: 185-201. https://doi.org/10.1016/j.jnca.2013.04.001

[5] Hammoudeh, M., and Robert N. (2015). "Adaptive routing in wireless sensor networks: QoS optimisation for enhanced application performance." Information Fusion, 22: 3-15. https://doi.org/10.1016/j.inffus.2013.02.005

[6] Lu, J., Wang, X., Zhang, L., \& Zhao, X. (2014). "Fuzzy random multi-objective optimization based routing for wireless sensor networks." Soft Computing, 18(5): 981-994. https://doi.org/10.1007/s00500-013-1119-2 
Paper-Routing Algorithm of Wireless Sensor Network and Robustness Analysis Based on Fuzzy Mat..

[7] Javidi, T., and Eric V.B. (2016). "Opportunistic Routing in Wireless Networks." Foundations and Trends ${ }^{\circledR}$ in Networking, 11(1-2): 1-137.

[8] Xiao-Chen, H., Wei-Jing, L., Min-Jie, X., Ning, Y., \& Xiao-Yue, R . "Energy balance and robustness adjustable topology control algorithm for wireless sensor networks." Acta Physica Sinica 64.8 (2015).

[9] Ozturk, C., Emrah H., and Dervis K. (2015). "A novel binary artificial bee colony algorithm based on genetic operators." Information Sciences, 297: 154-170. https://doi.org/10.1016/j.ins.2014.10.060

[10] Shelke, M., Tefera, G., Malhotra, A., \& Mahalle, P. (2016). "Fuzzy-based fault-tolerant low-energy adaptive clustering hierarchy routing protocol for wireless sensor network." International Journal of Wireless and Mobile Computing, 11(2: 117-123. https://doi.org/10.1504/IJWMC.2016.080183

[11] Gao, T., Song, J.Y., Zou, J.Y., Ding, J.H., Wang, D.Q., \& Jin, R.C. (2016). "An overview of performance trade-off mechanisms in routing protocol for green wireless sensor networks." Wireless Networks, 22(): 135-157.

[12] Ying, L., Qi, H., and Li W.Q. (2013). "Load-balanced clustering algorithm with distributed self-organization for wireless sensor networks." IEEE sensors journal, 13(5): 1498-1506. https://doi.org/10.1109/JSEN.2012.2227704

[13] Alanazi, A., and Khaled E. (2015). "Real-time QoS routing protocols in wireless multimedia sensor networks: Study and analysis." Sensors, 15(9): 22209-22233. https://doi.org/10.3390/s150922209

[14] Li, P., Yu, X., Xu, H., Qian, J., Dong, L., \& Nie, H. (2017). "Research on secure localization model based on trust valuation in wireless sensor networks." Security and Communication Networks 2017.

[15] Abhishek K., Sharma, R.K., Burnwal, A. (2015). "Energy Consumption Model in Wireless Ad-hoc Networks using Fuzzy Set Theory." Global Journal of Advanced Research, 2(2): 419-426.

[16] Kong, L., Pan, J.S., Snášel, V., Tsai, P.W., \& Sung, T.W. (2017). "An energy-aware routing protocol for wireless sensor network based on genetic algorithm." Telecommunication Systems, 2017: 1-13.

[17] Mary, S.A, Sahaaya, A., and Jasmine, B.G. (2016). "Fuzzy Logic Approach to Zone-Based Stable Cluster Head Election Protocol-Enhanced for Wireless Sensor Networks." KSII Transactions on Internet and Information Systems (TIIS), 10(4): 1692-1711.

[18] Mann, P.S., and Satvir, S. (2017). "Energy-Efficient Hierarchical Routing for Wireless Sensor Networks: A Swarm Intelligence Approach." Wireless Personal Communications, 92.(2): 785-805. https://doi.org/10.1007/s11277-016-3577-1

\section{Authors}

Ying Xiang received her Bachelor Degree in School of Mathematical Sciences, Nanjing Normal University, Nanjing, China in 2003 and the Master Degree in School of Mathematics, Southeast University, Nanjing, China in 2012. She is a lecturer working in Changzhou Vocational Institute of Mechatronic Technology, Basic Department of Culture, Changzhou 213164, China. She has published 12 papers. Her current research interests include mathematics pedagogy and Mathematical statistics.

Miaochao Chen received his Bachelor Degree in School of Mathematical Sciences, Capital Normal University, Beijing, China in 2004 and the Master Degree in 
School of Mathematics, Southeast University, Nanjing, China in 2012. He is a lecturer working at the School of Applied Mathematics, Chaohu University, Chaohu 238000, China.

Xiaohong Zhuang received her Bachelor Degree in School of Mathematical Sciences, Soochow University, Suzhou, China in 1986. She is an associate professor working at the Basic Department of Culture, Changzhou Vocational Institute of Mechatronic Technology, Changzhou 213164, China.

Xiaoxing Li received his Bachelor Degree in Automation College, Southeast University, Nanjing, China in 2002. He is a Mechanical and electrical manager working in Future Land Holdings Co. Ltd., Shanghai 200000, China.

Article submitted 25 October 2017. Published as resubmitted by the authors 27 November 2017. 